\title{
Case Report:Recombinant Tissue Plasminogen Activator Thrombolysis in Acute Ischemic Stroke First Case Report in Manipur
}

\author{
Dr Dipankar Paul ${ }^{1}$, Prof Th Suraj Singh ${ }^{2}$, Prof Th Bhimo Singh ${ }^{3}$, \\ Dr K Romeo Singh ${ }^{4}$ \\ ${ }^{1}$ (Department of Medicine, Regional Institute of Medical Science, Manipur, India) \\ ${ }^{2}$ (Department of Medicine, Regional Institute of Medical Science, Manipur, India) \\ ${ }^{3}$ (Department of Medicine, Regional Institute of Medical Science, Manipur, India) \\ ${ }^{4}$ (Department of Medicine, Regional Institute of Medical Science, Manipur, India)
}

\begin{abstract}
Cerebrovascular disease represents an enormous burden of disease and disability to mankind. Intravenous thrombolysis with recombinant tissue plasminogen activator(rtpa) within $3 \mathrm{hr}$ of symptomonset is currently approved for treatment of acute ischaemic stroke. Though it is widely used in western countries, the use of IV(intravenous) thrombolysis in peripherally located hospital is still a problem because for its short window period of $3 \mathrm{hr}$ of stroke onset.Here we are reporting the first case of IV thrombolysis in RIMS with recombinant tissue plasminogen activator(rtpa) recently. 78 years old man presented to us with right hemiparesis, power (1/5), global aphasia and altered sensorium. Patient presented to us at around one and half hour of stroke. At the time of presentation patient National institute of health stroke scale(NIHSS) score was 12 and Barthel index was O. CT head done show no infraction, patient was thrombolysedwith $50 \mathrm{mgActilys}$ (alteplase) within 30 minute of arrival to the hospital. Patient started improvement within 30 minute of starting thrombolysis, after $12 \mathrm{hr}$ of thrombolysis patient had power(4/5) with mild sensory aphasia. Repeat CT shows large left Middle cerebral artery(MCA) infract and CT angiography shows normal circle of willis.
\end{abstract}

Keywords: Acute ischaemic stroke, Alteplase, IV thrombolysis

\section{Introduction}

Cerebrovascular disease represents an enormous burden of disease and disability to mankind. The world health organization estimates that 15 million people world wide suffer a stroke each year[1]. For many decades intravenous thrombolysis have been delivered to treat acute thrombosis. Specifically the aim has been to recanalize the occluded artery and to restore perfusion to the brain that remain salvageable. IV thrombolysis for ischaemic stroke began its widespread adoption in the late 1990s after the publication of the national institute of neurological disorder and stroke study. Recombinant tissue plasminogen activator was approved by the food and drug administration in the mid 1990s for the rapid lysis of ischaemic stroke. The tissue plasminogen activator resulted in a clinically important and has continued to be the most important achievement in medical treatment of stroke[2]. Intravenous thrombolysis with recombinant tissue plasminogen activator within 3 hour of symptom onset is currently approved for the treatment of ischaemic stroke[3].

\section{Case}

A 78 year old hypertensive man presented to emergency with acute right hemiparesis. At the time of presentation his power was $1 / 5$ on the right side with global aphasia and altered sensorium. At the time of presentation patient NIHSSscore[4] was 12 and BARTHEL INDEX[5] was 0. CT was done shows no infraction. BP 132/86, PR 68/min, Glasgow coma scale E(3)M(6)V(1), Pupil normally reacting, Hemiplegia is incomplete right sided, Speech is global aphasia, Facial weakness absent, Tongue weakness absent, Plantar is extensor on the right side and other neurological examination are within normal limit. Chest, Cardiovascular, Abdomen examination are within normal limit.

\section{Course In The Hospital}

Our patient presented to us within one and half hour of stroke onset. As the patient presented within the window period, So after looking for the following contraindication likesymptoms rapidly improving, haemorrhagic stroke, head trauma within 3 month, major surgery within last 14 days, systolic blood pressure more than $185 \mathrm{mmhg}$, diastolic blood pressure more than $110 \mathrm{mmhg}$, subarachnoid haemorrhage, patient received treatment of anticoagulant in the past 48hour, arterial puncture at the non compressible site within 7 days, 
gastrointestinal haemorrhage within 2 days, INR more than 1.7, platelet count less than 100000,seizure at onset of stroke, serum glucose less than 50mg/dl and 400mg/dl[6]. We have started thrombolysis.

Within 30 minute of arrival to the hospital patient was thrombolysed with 50mg of Actilyse (alteplase), $10 \%$ of the dose was given as bolus and rest was given as a infusion over 1 hour. Patient had improvement within 30 minutes of start of thrombolysis. 12 hour after thrombolysis patient had power (4/5) with mild sensory aphasia. Check CT scan shows large left MCA infract and CT angiography shows normal circle of willis.

\subsection{CBC}

\section{Investigation}

Haemoglobin 10.9, TLC 7100( polymorphs72\%, lymphocytes 24\%, monocytes $2 \%$, eosinophil $2 \%$, basophil $0 \%$ ), Platelet 1.8 lac, ESR 40

4.2 KFT serum electrolyte

Serum creatinine 0.9, Urea 29, Na 129, K 4, CL 96

4.3 LFT

Total bilirubin 0.9, SGOT 52, SGPT 54, Total protein 6.1, Albumin 3.4, Globulin 2.7, ALP 84

4.4 LIPID PROFILE

Total cholesterol 220, Triglyceride 65, HDL 74, LDL 88, VLDL 34

4.5 NCCT BRAIN

Left MCA territory infract involving parieto temporal and occipital lobes subcortical area no midline shift 4.6 CT ANGIOGRAPHY

Hypoplastic left posterior cerebral artery with prominent left posterior communicating artery

4.7 ECHOCARDIOGRAPHY

EF 59\%, mild MR, mitral annular calcification, trivial TR, clot not visible

4.8 ECG

AF heart rate $58 / \mathrm{min}$

4.9 RBS 87mg/dl, URINE ROUTINE epithelial cell 2-3/hpf, HIV negative, PT 12sec, INR 1.6, URINE CULTURE sterile, HBsAG and HCV negative, Sputum for AFB and Malignant cells negative, Thyroid profile normal, Chest x ray normal

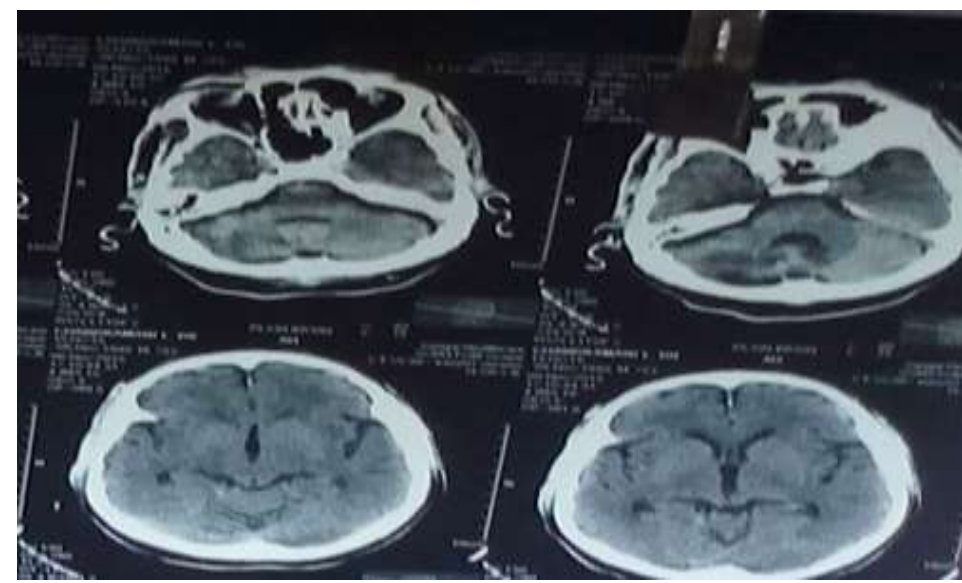

fig 1: ct scan before thrombolysis

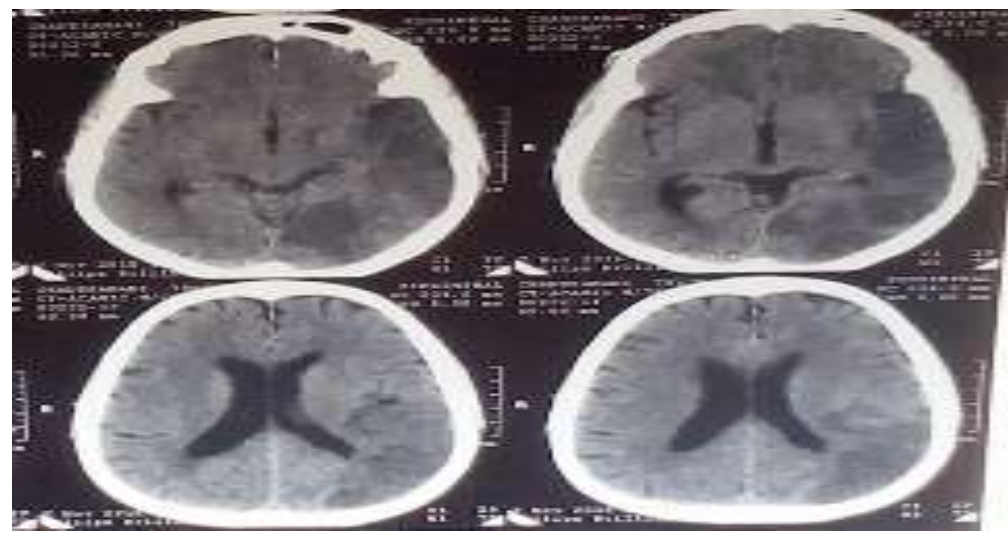

fig 2: ct scan post thrombolysis 


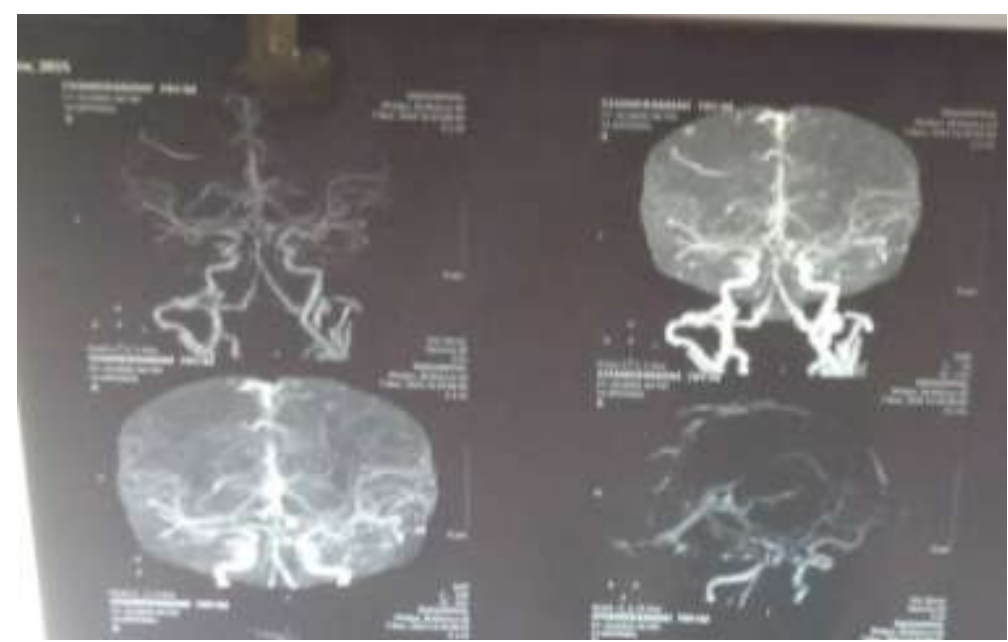

fig 3: ct angiography

\section{Discussion}

The patient presented to us with right hemiparesis with altered sensorium suggesting major stroke with possible main stem MCA (middle cerebral artery) block. Though at presentation CT scan was normal repeat CT scan after post thrombolysis was showing MCA infract with no ICH (intracranial haemorrhage ). CT angiography showed no thrombosis and stenosis in ICA (internal carotid artery) or MCA( middle cerebral artery). Which proved to be successful thrombolysis. In spite of residual infract patient has only residual sensory aphasia at the time of discharge with complete improvement in hemiparesis.

\section{References}

[1]. Mackay JMG, the atlas of heart disease and stroke (London, world stroke organization, 2004).

[2]. Aspland KSB, Peltonem M, From the twentieth to the twenty first century a public health perspective on stroke, in Ginsberg $\mathrm{MD}(\mathrm{Ed})$,cerebrovascular diseasepathophysiology diagnosis and treatment(Maldem mass: Blackwell science, 1998).

[3]. Sylaja PN, Demchuk AM, Intravenous thrombolytic therapy in acute ischemic stroke : The art and science of treatment decision making, Annals of Indian academy of neurology,11(5), 2008, 24-29.

[4]. NIH stroke scale, available at :http://www.ninds.nih.gov/doctors/nih stroke scale, Accessed on july 21, 2016.

[5]. Mahoney FI, Barthel D, Functional evaluation the Barthel index,Maryland state medical journal, 14, 1965, 56-61.

[6]. AHA acute stroke treatment guidelines 2007, stroke, 38(5), 2007,1655. 